\title{
Analysis of Microvascular Blood Flow and Oxygenation: Discrimination between two Haemodynamic Steady States using Nonlinear Measures and Multiscale Analysis
}

\author{
Marjola Thanaj ${ }^{a}$, Andrew J. Chipperfield ${ }^{a}$ and Geraldine F. Clough ${ }^{b}$ \\ a Bioengineering Science Group, Faculty of Engineering and the Environment, University of Southampton, \\ Highfield, Southampton, SO17 1BJ, UK (corresponding email: m.thanaj@soton.ac.uk) \\ ${ }^{b}$ Human Development \& Health, Faculty of Medicine, University of Southampton, Southampton, UK
}

\begin{abstract}
Objective: This study investigates the feasibility of the use of nonlinear complexity methods as a tool to identify altered microvascular function often associated with pathological conditions. We evaluate the efficacy of multiscale nonlinear complexity methods to account for the multiple timescales of processes modulating microvascular network perfusion. Methods: Microvascular blood flux (BF) and oxygenation ( $\mathrm{OXY}: \mathrm{oxyHb}$, deoxy $\mathrm{Hb}$, total $\mathrm{Hb}$ and $\mathrm{SO}_{2} \%$ ) signals were recorded simultaneously at the same site, from the skin of 15 healthy young male volunteers using combined laser Doppler fluximetry (LDF) and white light spectroscopy. Skin temperature was clamped at $33^{\circ} \mathrm{C}$ prior to warming to $43^{\circ} \mathrm{C}$ to generate a local thermal hyperaemia (LTH). Conventional and multiscale variants of sample entropy (SampEn) were used to quantify signal regularity and Lempel and Ziv (LZ) and effort to compress (ETC) to determine complexity. Results: SampEn showed a decrease in entropy during LTH in $B F(p=0.007)$ and oxygenated haemoglobin $(o x y H b)(p=0.029)$. Complexity analysis using $L Z$ and ETC also showed a significant reduction in complexity of $B F(L Z, p=0.003$; $E T C, p=0.002)$ and oxyHb $(p<0.001$, for both) with LTH. Multiscale complexity methods were better able to discriminate between haemodynamic states $(p<0.001)$ than conventional ones over multiple time-scales. Conclusion: Our findings show that there is a good discrimination in complexity of both $\mathrm{BF}$ and oxyHb signals between two haemodynamic steady states which is consistent across multiple scales. Significance: Complexity-based and multiscale-based analysis of BF and OXY signals can identify different microvascular functional states and thus has potential for clinical application in the prognosis and the diagnosis of pathophysiological conditions such as microvascular dysfunction observed in nonalcoholic fatty liver disease and type 2 diabetes.
\end{abstract}

Keywords: Blood flow, tissue oxygenation, sample entropy, Lempel and Ziv complexity, effort to compress complexity, multiscale analysis.

\section{Introduction}

The maintenance of an adequate blood flow through a microvascular network, sufficient to meet the metabolic demands of the tissue, is dependent on local endothelial, metabolic, myogenic and neural vaso-mechanisms that determine vascular tone and thus temporal and spatial flow patterns within the network [1]. Recently, Frisbee et al. [2] have shown attenuation of these flow patterns using chaotic network attractor analysis in an animal model of cardio-metabolic disease. They have argued that the consequent loss of physiological information content may contribute to disease risk $[3,4]$.

Time and frequency domain analysis and the contribution of spectral properties in frequency domains are the techniques most frequently applied to biosignals [5-7], including those derived from blood flow through the superficial dermal microvasculature [8-10]. The frequency and power of local oscillations that contribute to the total blood flow motion have been studied by many research groups $[8,11,12]$. They contain the cardiac $(\sim 0.6-1.6 \mathrm{~Hz})$ and respiratory $(\sim 0.15-0.4 \mathrm{~Hz})$ activity followed 
by oscillations arising from local myogenic vasomotor activity $(\sim 0.06-0.15 \mathrm{~Hz})$. Two additional components that occur in lower frequencies are the neurogenic $(\sim 0.02-0.06 \mathrm{~Hz})$ and endothelial $(\sim$ $0.0095-0.02 \mathrm{~Hz}$ ) activity. It has been widely argued that time frequency analysis of the low frequency periodic oscillations in microvascular blood flux (BF) signals obtained using for example laser Doppler fluximetry (LDF) can provide non-invasive, mechanistic information on microvascular control $[8,13]$.

The regularity and the randomness of physiological signals has been explored using nonlinear methods such as entropy and complexity techniques, respectively, which are well suited for the analysis of short length signals such as ECG and respiratory flow signals [9, 14-17]. Lempel-Ziv (LZ) complexity analysis has been applied to skin microvascular BF signals in humans [10] and in animal models, $[9,18]$ in differing haemodynamic states. These studies have demonstrated clear differences in LZ complexity between haemodynamic states. However, the relationship between the nonlinear dynamics of the BF signal and their impact on microvascular function remain to be clarified and the potential for complexity analysis as a diagnostic tool determined.

Conventional nonlinear methods have the drawback that they can only study the behaviour at one scale. Multiscale entropy algorithms have been used to quantify the complexity of the biological signals across multiple spatial and temporal scales $[14,19,20]$. Multiscale entropy approaches have shown good discrimination between the cardiac signals of young, elderly and subjects with heart failure [3], and multiscale LZ complexity demonstrating a $86 \%$ classification accuracy of the information content in the EEG signals of control and depressive groups [21]. Further, wavelet transform and multiscale complexity analysis of temperature signals has been used to classify risk in patients with sepsis [22]. Together these studies evidence the potential for such approaches in the characterisation of the flexibility/responsiveness of the microvasculature, particularly in individuals at risk of developing or with cardio-metabolic disease.

In this study, our aim was to investigate the information content of BF and OXY signals derived from the microvasculature in two stable haemodynamic steady states: at rest with the local skin temperature clamped at $33^{\circ} \mathrm{C}$ and during vasodilation induced through local thermal hyperaemia (LTH) at $43^{\circ} \mathrm{C}$, using both conventional and multiscale techniques. The local thermal warming skin test is routinely used in clinical applications to test dilator responses [23], as other perturbation techniques such as post-occlusive reactive hyperaemia do not induce an enduring state change which was required in this study to test the efficacy of the complexity methods. Here, we first explore the changes in complexity of the microcirculatory dynamics using conventional sample entropy (SampEn), LZ and effort to compress (ETC) complexity methods. To understand the effect of scale on these nonlinear metrics and their efficacy in classifying these haemodynamic steady states the multiscale sample entropy (MSE), multiscale Lempel and Ziv (MSLZ) complexity and multiscale effort to compress (METC) methods are then evaluated.

We hypothesise that complexity-based and multiscale-based analysis of blood flux and tissue oxygenation signals derived from the skin of healthy individuals under two imposed haemodynamic steady states will enable the characterisation of the flexibility/responsiveness of a microvascular network and thus has potential for clinical application in the prognosis and the diagnosis of pathophysiological conditions.

\section{Materials and Methods}

\subsection{Study Design}


The study was conducted on 15 healthy male participants, age $29.2 \pm 8.1 \mathrm{y}$ (mean \pm SD). All participants were asked to refrain from caffeine-containing drinks for at least two hours before the measurement and to avoid exercise on the day of study. None of the participants were taking any medications. All measurements were made in a temperature controlled room $\left(23.0-23.5^{\circ} \mathrm{C}\right)$ and all participants were acclimatized for at least 20 minutes before measurements were taken. Measurements were made with the participant sitting comfortably in a reclining blood infusion chair with their arm supported at heart level [10]. The study was approved by Research Ethics Committee of University of Southampton and Southampton General Hospital (REC Number: SOMSEC091.10; RHMMED0992). The study was performed in accordance with standards set by the Declaration of Helsinki. All participants gave informed written consent. All data supporting this study are openly available from the University of Southampton repository at http://doi.org/10.5258/SOTON/D0343.

\subsection{Acquisition of Laser Doppler and OXY Signals}

Skin microvascular BF and oxygenation (OXY) signals were recorded simultaneously using a combined laser Doppler flowmetry and white light reflectance probe (Moor CP7-1000 blunt needle probe, Moor Instruments Ltd, Axminster, UK) using a single point $785 \mathrm{~nm}, 1 \mathrm{~mW}$ low power red laser light source (moorVMS-LDF2, Moor Instruments Ltd, UK) and 400-700 nm, $<6 \mathrm{~mW}$ white light source (moorVMS-OXY, Moor Instruments Ltd, UK). The probe was mounted in a MoorVHP1 skin heating block controlled by the MoorVMS-HEAT skin heater. Skin temperature was measured by a miniaturised negative temperature coefficient thermistor built into the heating block controlling skin warming with a precision of $\pm 0.1^{\circ} \mathrm{C}$ and resolution of $0.1^{\circ} \mathrm{C}$. As shown in Fig. 1 the heating block and probe were placed on the ventral surface of the non-dominant forearm using a double-sided sticky $\mathrm{O}$ ring, approximately $10 \mathrm{~cm}$ from the wrist and avoiding visible veins.

The $\mathrm{BF}$ and $\mathrm{OXY}$ recordings were obtained in two haemodynamic steady states, with the heating block clamped at $33^{\circ} \mathrm{C}$ and then at $43^{\circ} \mathrm{C}$ during LTH. To study the signals during these steady states, the data were divided into segments of 10 minutes duration. All recordings were captured at a sampling rate of $40 \mathrm{~Hz}$ using the manufacturer's software (MoorSoft). Fig. 2 illustrates the BF, OXY and the temperature outputs of the combined LDF/OXY probe recorded and the selection of the 10 minutes segments marked as grey at $33^{\circ} \mathrm{C}$ and at $43^{\circ} \mathrm{C}$, respectively. These segments were selected so as to minimise any transitional effects arising during warming and to be free of movement artefacts. Data were exported to Matlab (R2016b, Mathworks, UK) for pre-processing and analysis. The truncated data could then be analysed and calculations made for: (i) the entropy and the complexity analysis and (ii) the multiscale analysis. The parameters obtained were BF in perfusion units (PU), oxygenated haemoglobin (oxyHb), deoxygenated haemoglobin (deoxy $\mathrm{Hb}$ ), total haemoglobin (total $\mathrm{Hb}$ $=0 x y \mathrm{Hb}+$ deoxy $\mathrm{Hb}$ ) in arbitrary units $(\mathrm{AU})$ and tissue oxygen saturation $\left(\mathrm{SO}_{2}\right) . \mathrm{SO}_{2}(\%)$ is derived from the relationship $\mathrm{SO}_{2}=($ oxy Hb/total $\mathrm{Hb}) \times 100 \%$ [24]. We elected to focus on the oxyHb output as the prime OXY signal for the entropy and complexity analysis as suggested by our previous studies [10]. 


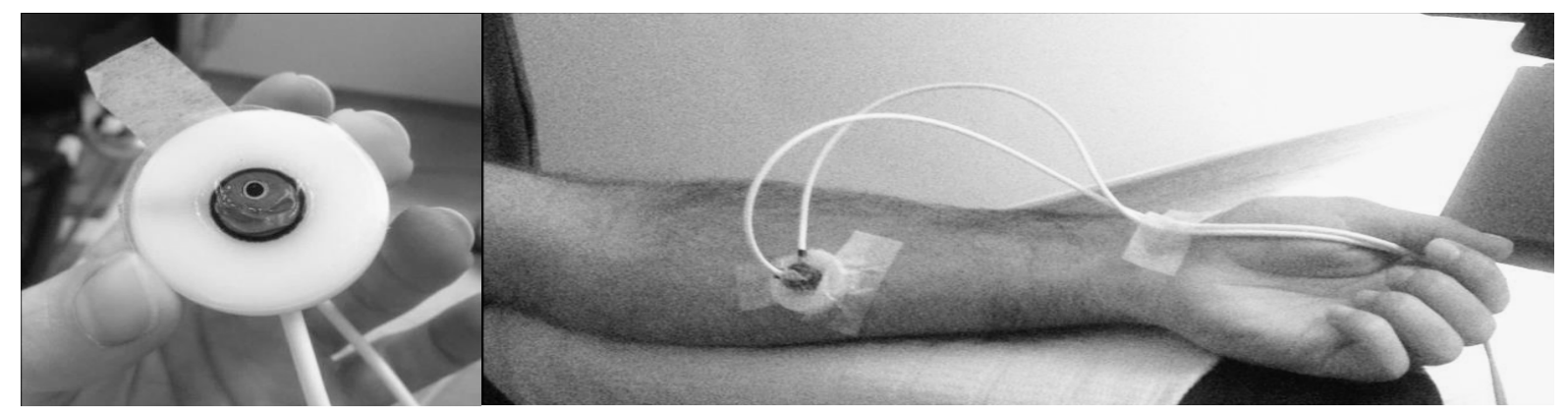

Fig. 1 The combined LDF and WLS probe inserted into a heating disc (left) and attached to the skin (right).

\subsection{Pre-processing}

Before estimating signal complexity, it is necessary to perform some pre-processing of the raw data. The data segments were filtered using a low pass finite impulse response (FIR) filter with $2 \mathrm{~Hz}$ cut-off frequency, to attenuate high frequencies beyond the known range of microvascular oscillation, $0.0095-1.6 \mathrm{~Hz}[8,25]$. The data were then detrended by removing the mean. A stationarity test was performed on the processed time series to examine whether the trend was removed and our data had a consistent variance over time. This pre-processing ensures that the analysis of the signals was not biased by noise or components outside the range of interest.

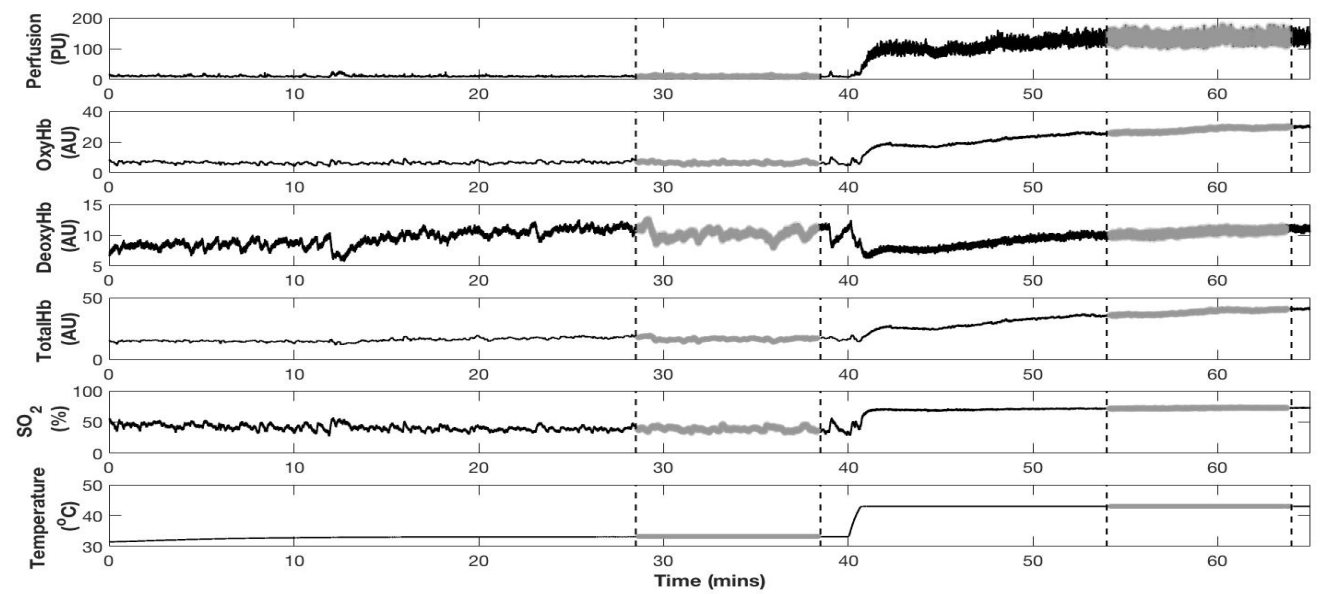

Fig. 2. Selection of the 10 minutes segments (grey trace) for the data analysis at $33^{\circ} \mathrm{C}$ and $43^{\circ} \mathrm{C}$. Blood flow in arbitrary perfusion units (PU), oxygenated haemoglobin (oxyHb), deoxygenated haemoglobin (deoxyHb), total haemoglobin (total $\mathrm{Hb}=0 x y \mathrm{Hb}+$ deoxy $\mathrm{Hb}$ ), all in arbitrary units ( $\mathrm{AU}$ ), tissue oxygen saturation $\left(\mathrm{SO}_{2}=(\mathrm{oxyHb} /\right.$ total $\left.\mathrm{Hb}) \times 100 \%\right)$ and temperature $\left({ }^{\circ} \mathrm{C}\right)$ plots were obtained from one individual.

\subsection{Nonlinear methods}

Binary Conversion: The nonlinear methods used here are based on the information content of a finite time series to calculate the randomness of its binary representation [26]. From previous studies $[27,28]$, it was reported that the binary conversion is sufficient to estimate the complexity in 
biomedical signals. Therefore, before calculating the complexity, the signal has to be converted into a sequence of zeros and ones. Many researchers [9, 28, 29], convert the time series into 0-1 sequence by comparing each value with a threshold, which is usually set as the mean or the median value of the sequence, and replacing the value with zero if it is lower than the threshold and one if it is higher. In this work, a different approach is employed to maximise the information contained in the binary sequence. As suggested by Yang et al. [30], a straightforward way to maintain the important characteristics of the dynamics of the original physiologic signal is by using the increase and decrease encoding method, which will be referred here as delta encoding.

Let $x=x_{1} x_{2} \cdots x_{i}$ denote a time series with length $i$. Each element in the time series is compared with the previous element. If the value of the current element in the time series is increased compared to the previous value the corresponding element of the binary encoded sequence is set as 1 . Otherwise, if it is decreased, then the value in the encoded sequence is denoted as 0 . Thus, the time series is transformed into 0-1 sequence $s=s_{1} s_{2} \cdots s_{n}$ with $s_{n}$ expressed as.

$$
s_{n}= \begin{cases}0, & \text { if } x_{n} \leq x_{n-1} \\ 1, & \text { if } x_{n}>x_{n-1} .\end{cases}
$$

Now the binary sequence will have a length $n$, where $2 \leq n \leq i$, which is different from the length $i$ in time series, referred as $n$-bit. Each $n$-bit correspond to a "word" of the binary sequence $s$ and every $n$-bit represents the dynamics of the time series $x$. The nonlinear methods employed here are estimated as follows:

Sample Entropy: Sample entropy (SampEn) provides an applicable finite sequence formulation that discriminates the data sets by a measure of randomness, from totally regular to completely random. SampEn assigns non-negative patterns in time series, with larger values of entropy corresponding to more irregularity and smaller values corresponding to more regularity in the data. The regularity of the signal can be measured with the SampEn, by defining how often a short time series is repeated. Sample Entropy is described as a refinement of another similar measure, Approximate Entropy (ApEn) that was introduced by Pincus [31]. Approximate entropy has proved to be a sufficient measure of the regularity for many signals, however, it is a biased measurement towards regularity and it is dependent on the length of the signal. To overcome these issues with ApEn, SampEn was developed by Richman and Moorman [32].

In order to measure SampEn the time series is converted into a binary sequence using the delta encoding, then two parameters are defined, $m$, a positive integer that denotes the length of the compared runs and $r$, a positive real number that denotes the tolerance window. SampEn measures the logarithmic likelihood that runs of samples that are close for $m$ continuous observations that remain close (within the same tolerance window $r$ ) on subsequent incremental comparisons. Given a binary sequence of $N$ data $s(n)=s(1), s(2), \ldots, s(N)$, according to Richman and Moorman [32], for the time series analysis and Scoric et al. [33], for the analysis of the binary sequence, the sequence is computed to estimate the binary sample entropy. However, the SampEn sequence is binary and thus is limited diversity. For that reason, the number of elements of a set of different $m$-bits integers is equal to $2^{m}$ and the binary SampEn is calculated as follows:

- To each of the binary sequence elements $S(i)=[s(i), s(i+1 * \tau), \ldots, s(i+m * \tau], i=1 \ldots N-$ $m * \tau$, a decimal counterpart $c$ can be evaluated as:

$$
c=\sum_{k=0}^{m-1} s_{i+k * \tau} * 2^{k}
$$


- Define the distance between $S(i)$ and $S(j)$ as:

$$
d[S(i), S(j)]=\max _{k=0,1, \ldots, m-1}\left|s(i+k * \tau) * 2^{k}-s(j+k * \tau) * 2^{k}\right|
$$

- Denote $N^{m}(l)=$ number of $S(j)(j=1 \ldots N-m * \tau$ within $r$ of $S(i)(i=1 \ldots N-m * \tau)$, such that $d[S(i), S(j)] \leq r$ and defined for each $i, 0 \leq i \leq N-m * \tau$,

$$
C_{i}^{m}(r)=N^{m}(i) /(N-m * \tau)
$$

- Next, define:

$$
\Phi^{m}(r, N, \tau)=(N-m * \tau)^{-1} \sum_{i=1}^{N-m * \tau} C_{i}^{m}(\mathrm{r})
$$

- By increasing the run length, $C_{l}^{m+1}(r)$ and $\Phi^{m+1}(r, N, \tau)$ are counted. So, from a given $N$ data points, SampEn will be:

$$
\operatorname{SampEn}(m, r, N, \tau)=-\ln \frac{\Phi^{m+1}(r, N, \tau)}{\Phi^{m}(r, N, \tau)}
$$

Sample entropy requires the parameters $m, r$, and $\tau$ in order to provide meaningful results in the estimation of the entropy. The choice of these parameters varies for binary sequences. According to the literature [31-33], the parameters for the binary sample entropy estimation to effectively discriminate two data would be better set as $m \geq 2, r<1$ or 1 and $\tau \geq 1$. The $m$ parameter can be estimated using the false nearest neighbours method [34]. However, with experimental data problems, such as drifts, can occur so visual inspection can be also used to estimate the most suitable $m$ as well as $r$ parameters for analysis. The time delay $\tau$ can be obtained using either the autocorrelation function or the mutual information analysis when the correlation or averaged mutual information between the two instances $i$ and $i+\tau$ reaches its first relative minimum [34-36]. The parameters used in this study that produce satisfactory results after a visual inspection of SampEn for binary sequences of both short and large lengths, were set as $m=2, r<1$ and $\tau=1$. An interpretive example on physiological data for the choice for the parameters of the binary SampEn is shown in the supplemental figures 1 and 2 .

Lempel and Ziv Complexity: The LZ complexity has been used to estimate and quantify symbolic sequences converted from a time series $[9,28,37]$. It is a method of quantifying the information present in a signal or sequence by estimating the number of production processes (unique subsequences) contained in the data. Here, to calculate the $L Z$ complexity, the signal was first converted into a binary sequence using the delta encoding procedure. The sequence was then parsed from left to right and the complexity increases by one unit when a new sub-sequence of continuous symbols was encountered. The LZ algorithm can be described as follows [38]:

- Let $S=s_{1} s_{2} \cdots s_{n}$ be a string that denotes a binary sequence with a finite length $n$ that starts at the $i$ position and finishes at the $j$ position. So, when $i \leq j$ then $S(i, j)=\left\{s_{1} s_{i+1} \cdots s_{j}\right\}$ and when $i>j$ then $S(i, j)=\{\}$, a sequence with zero length is set.

- A notation $v(S)$ is introduced which denotes the vocabulary of the sequence $S$ and corresponds to the set of "words" that can be reconstructed from $S$. For example, if $S=010$, the vocabulary $v(S)$ will represent all the possible "words" that can be generated from the sequence, thus $v(S)=$ $\{0,1,01,10,010\}$. The vocabulary $v(S)$ is then compared with every bit $i$ of the binary sequence $S(i, j)$ which is up to $j-1$, so $i=1,2,3 \ldots n, j>i$. 
- Let $Q$ denote the prefix of $S$ that is used to determine if this term is included in the substring of $S$, so $Q$ can be obtained by copying the "words" of $S$. Then, set $\pi$ as the procedure to delete the last "word" of the sequence $S$, so $Q$ is a subsequence of the vocabulary $v(S Q \pi)$. A sequence $S$ is called reproducible, if the incoming bit is present in $v(S Q \pi)$ and producible if is not present and therefore a dot $\cdot$ is placed after the sub-sequence to indicate the end of a new component.

- To reconstruct the sequence $S$, it is essential to have a production process called production history of $S, H(S)=S\left(1, h_{1}\right) S\left(h_{1}+1, h_{2}\right) \ldots S\left(h_{m-1}+1, h_{m}\right)$, where $m$ are the "words" of the history and for $i=1,2 \ldots m, H_{i}(S)=S\left(h_{i-1}+1, h_{i}\right)$, where $h_{i}$ are the components of the history $H(S)$. So, for example, let a binary sequence $S=\{010100110110\}$, then the vocabulary $v(S)=$ $\{0,1,01,10,010 \ldots\}$ the sequence $H(S)$ which is the original sequence separated into components with dots by parsing the sequence $S$ from left to right, will be $H(S)=0 \cdot 1 \cdot 0100 \cdot 11 \cdot 0110$.

In order to define the complexity $c(S)$ of a sequence $S$, let denote $c_{H}(S)$ the least number of the components generated form the history $H(S)$ so,

$$
c(S)=\min \left\{c_{H}(S)\right\}
$$

In this example, the number of the components of the production history $H(S)$ are 5 and therefore, the complexity $c(S)$ is 5 .

According to Lempel and Ziv [38], for every sequence $S$ with a length $n$, the length of the production process of the sequence, $c(S)$, will be: $c(S)<\frac{n}{\left(1-\varepsilon_{n}\right) \cdot \log _{a}(n)}$, where $a$ is the base of the logarithm and $\varepsilon_{n}$ is a small quantity that tends to be equal to zero when the length $n$ of the sequence is large. So, for a binary sequence with $a=2$, the normalized complexity $C(S)$ will now be:

$$
C(S)=\frac{c(S)}{\frac{n}{\log _{2}(n)}}
$$

Thus, it is justified that a large length $n$ represents more complexity. In this work, the normalized version of the LZ complexity is used for convenience as it is independent of the length of the sequence and is suitable for comparing sequences with different lengths.

Effort to Compress Complexity: A similar complexity method based on the lossless compression algorithm known as Non-Sequential Recursive Pair Substitution (NSRPS) [39] is the Effort to compress (ETC) complexity [40]. Like the LZ complexity measure, the time series first has to be converted to a symbolic sequence using the delta encoding. So, let a binary sequence $S=\{010100110110\}$, here the algorithm differs from LZ complexity by detecting the reoccurrencing patterns directly and can be described as follows:

- Define the pair of symbols with the maximum occurrence and replace all its non-overlapping occurrences with a new symbol, at each iteration. This procedure is repeated and is dependent on NSRPS which estimates the entropy for each operation that the algorithm obtains, providing a new sequence $S_{\text {new }_{N}}, N=1 \ldots$ number of iterations, until it reaches a constant sequence.

- Now, the input sequence $S$ is first converted into $S_{\text {new }_{1}}=\{22021210\}$ considering that the pair " 01 " has maximum occurrences compared to pairs "00", "10" and "11".

- In the next iteration, $S_{n e w_{1}}$ is converted into $S_{n e w_{2}}=\{220330\}$ considering that the pair "21" has maximum occurrences. 
- The algorithm continues in this way until there is no pair left to replace and the converted sequence becomes a constant sequence.

In this example, the algorithm converts the given sequence $S$ as: $S=\{010100110110\} \rightarrow$ $S_{\text {new }_{1}}=\{22021210\} \rightarrow S_{\text {new }_{2}}=\{220330\} \rightarrow S_{\text {new }_{3}}=\{40330\} \rightarrow S_{\text {new }_{4}}=\{5330\} \rightarrow S_{\text {new }_{5}}=$ $\{630\} \rightarrow S_{\text {new }_{6}}=\{70\} \rightarrow S_{\text {new }_{7}}=\{8\}$.

So, according to Nagaraj et al. [40], the ETC complexity measure is defined as:

$$
N \rightarrow \text { Number of iterations of NSPRS algorithm for entropy } \rightarrow \text { zero }
$$

More specifically, $N$ is the number of iterations, of NSPRS algorithm, required for the given sequence to be transformed to a constant sequence with zero entropy. In this example, $N$ is 7 and the normalized measure is computed as: $\frac{N}{L-1}, 0 \leq N-1 \leq 1$, with $L=$ length of the sequence. In this work, the normalized measure was used.

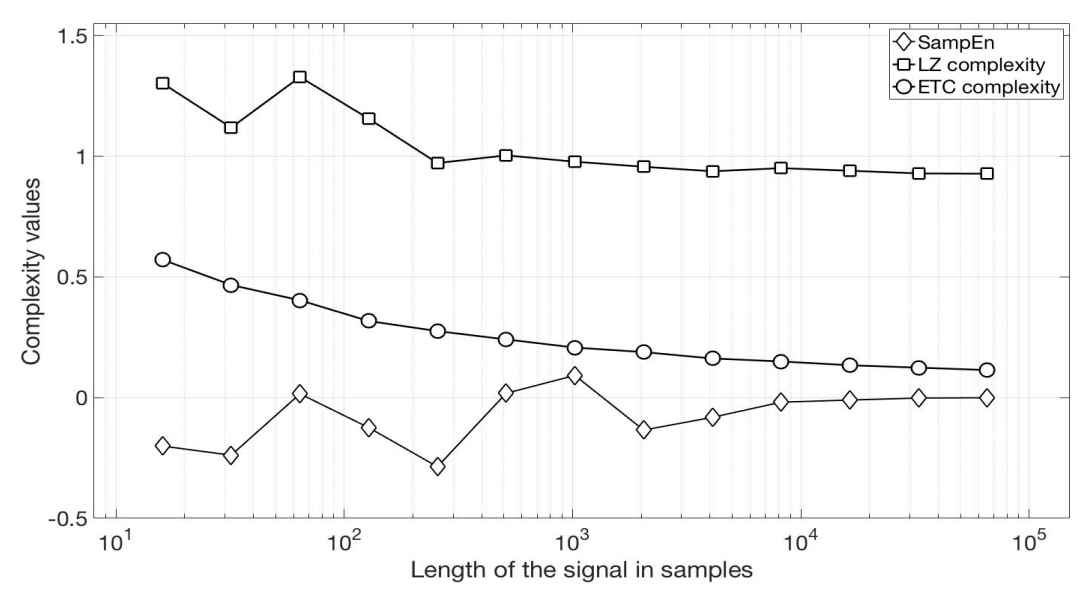

Fig. 3 Effect of the length of a random signal on binary SampEn, LZ and ETC. Binary SampEn, LZ and ETC complexity $\left(-\nabla_{-},-\mathrm{O}\right.$ - and - - markers, respectively) do not give any reasonable values of complexity for signals $<500$ samples.

In order to examine the implementation of the nonlinear methods through various signal lengths, we first applied the binary SampEn, LZ and ETC complexity to normally distributed random (generated from Matlab using the function "randn") signals with different length (Fig. 3). For short signals, binary SampEn, LZ and ETC give results that are not meaningful for the estimation of complexity. Only values for length higher than 500 samples provide a reasonable estimate of the complexity of the sequence. As shown from Fig. 3 there is no particular reason for choosing one of the nonlinear methods for analysis over the others as all these methods produce consistent values when the signal has more than 1000 samples. However, this needs to be tested in complex physiological signals in order to estimate the performance of these measures in various lengths. From this test, we have obtained useful information on the decision of the minimum length of the signal used for a sufficient nonlinear analysis.

Multiscale Analysis: Entropy and complexity methods are used to analyse signals on a single scale, however, when applying these methods to signals arising in complex physiological systems it is 
important to take into account the multiple time scales of the underlying processes modulating the signal. To examine if a signal is consistent across multiple spatial and temporal scales and does not change with different sampling frequencies, it is a necessity to measure the complexity of the time series in multiple time scales. Costa et al. [19], proposed the Multiscale Entropy (MSE) technique for analysing biological signals using a coarse-graining method that resamples the original signal by reducing the scale of the time series and then determining the sample entropy for each scale step. Thus, coarse graining is a method, which alters the sampling frequency of the signal by a scale factor, $\tau$, which defines the number of the sampling levels. So, for a time series $\left\{x_{1}, \ldots, x_{N}\right\}$, the coarsegrained time series, $y^{\tau}$, will be:

$$
y_{i}^{\tau}=\frac{1}{\tau} \sum_{i=(i-1) \tau+1}^{i \tau} x_{j}, 1 \leq i \leq N / \tau .
$$

For a scale factor of one the time series $y^{1}$ is the original signal. So, the length of each time series $\left\{y^{\tau}\right\}$ is the equal to the length of the original signal divided by the scale factor, $\tau$. A straightforward way to illustrate the coarse-graining procedure is shown in Fig. 4, using a skin BF signal coarsegrained at multiple scale lengths, by averaging the data points of the BF signal. It is also shown that by scaling the original signal, many of the features were expanded but the information of the signal has remained.

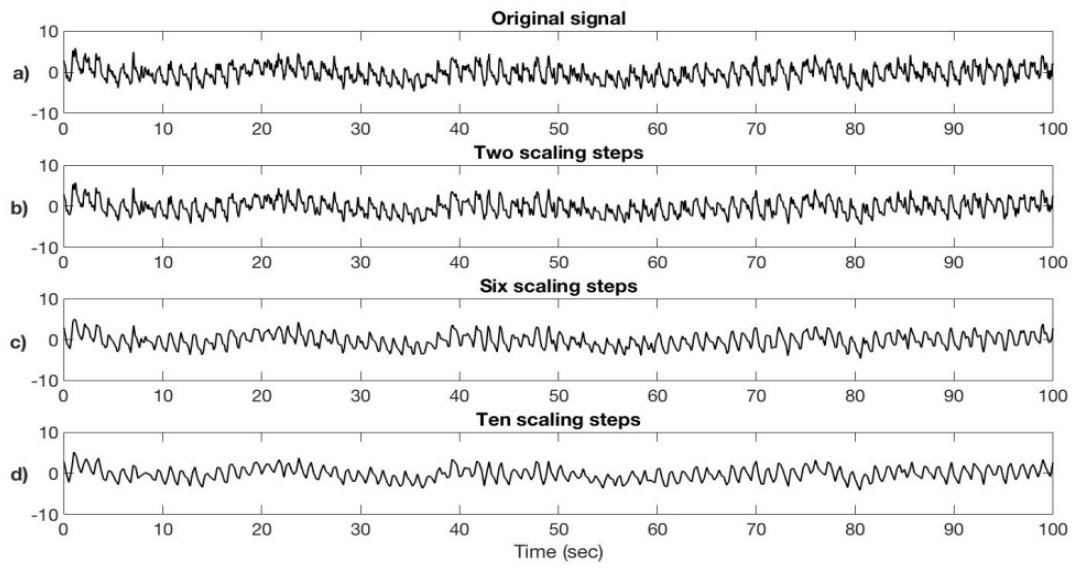

Fig. 4 A skin BF signal coarse-grained at multiple scale lengths. a) The original signal, b) the signal down scaled two times, c) the signal down scaled six times, d) the signal down scaled ten times.

\subsection{Analysis procedure}

Here, Sample entropy, LZ complexity and ETC complexity were applied to 10-minutes (24000 samples) of skin $\mathrm{BF}$ and oxyHb signals $\left(f_{s}=40 \mathrm{~Hz}\right)$. The signals were, then divided in 15 epochs of length 40 seconds, which is a consistent with previous work $[9,10]$, and the complexity calculated using each method, for each epoch. Additionally, the multiscale analysis was applied to the BF and oxyHb signals, in order to get information in multiple scales. At the coarse-grain of 24 scales, the shortest signal has 1000 samples, which is sufficient for analysis as shown in Fig. 3 and is consistent with other literature $[14,19,32]$. Then, SampEn, LZ and the ETC complexity were calculated for each coarse-grained sequence as a function of the scale factor, $\tau$. We call these procedures multiscale entropy (MSE), multiscale Lempel and Ziv complexity (MSLZ) and multiscale effort to compress complexity (METC). 


\subsection{Statistical analysis}

Statistical analysis was performed in IBM SPSS statistics 22 (IBM United Kingdom) and Microsoft Excel 2010 (Microsoft Corporation, US). Data were tested for normal distribution using the D'Agostino \& Pearson omnibus normality test. Data are presented as mean and mean standard error. Normally distributed data were compared using the Student $t$-test for paired data to compare mean values of normally distributed data. P-values less than 0.05 were taken to indicate statistical significance.

\section{Results}

The nonlinearity of the $\mathrm{BF}$ and oxyHb signals at the two haemodynamic steady states of $33^{\circ} \mathrm{C}$ and $43^{\circ} \mathrm{C}$ was computed for all methods for the 15 individuals as shown in Fig. 5 and 6 , respectively. Both signals presented lower values of complexity during LTH as compared with those at $33^{\circ} \mathrm{C}$. Table 1 gives summary statistics for all complexity methods applied in $\mathrm{BF}$ and oxyHb signals at both temperatures over the 15 epochs.
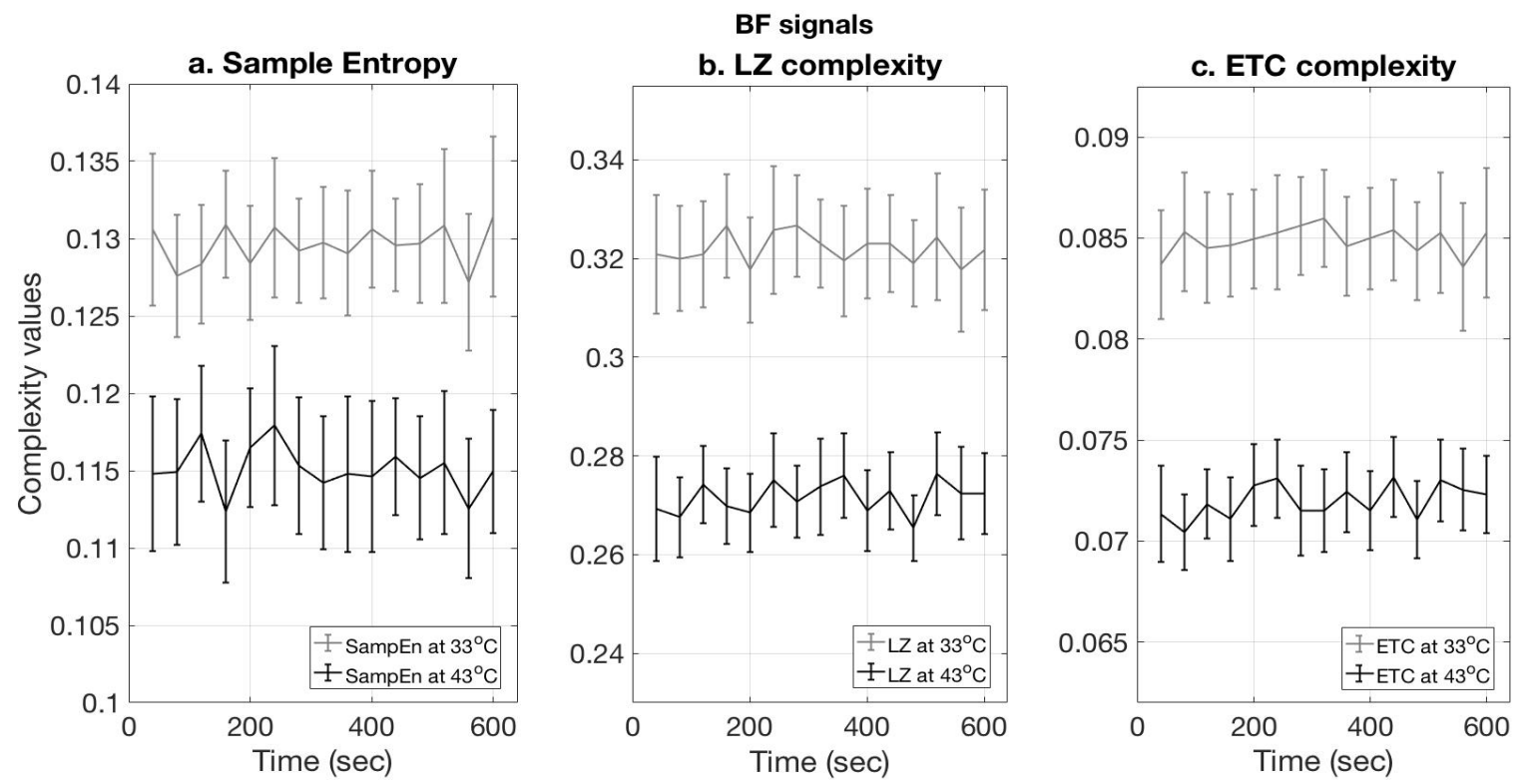

Fig. 5 Changes in the complexity of the blood flow signals for all 15 volunteers, in two haemodynamic steady states at $33^{\circ} \mathrm{C}$ (grey) and at $43^{\circ} \mathrm{C}$ (black). a) Sample Entropy, b) LZ complexity, c) Effort to compress complexity. Values are presented as means \pm standard errors of the mean (SEM).

Overall the SampEn index (mean value across the 15 epochs for each participant) showed a decrease in both BF and oxyHb signals with LTH. Likewise, the $L Z$ index showed a reduction in the complexity during local heating, in the BF signals, between the two haemodynamic steady states. 

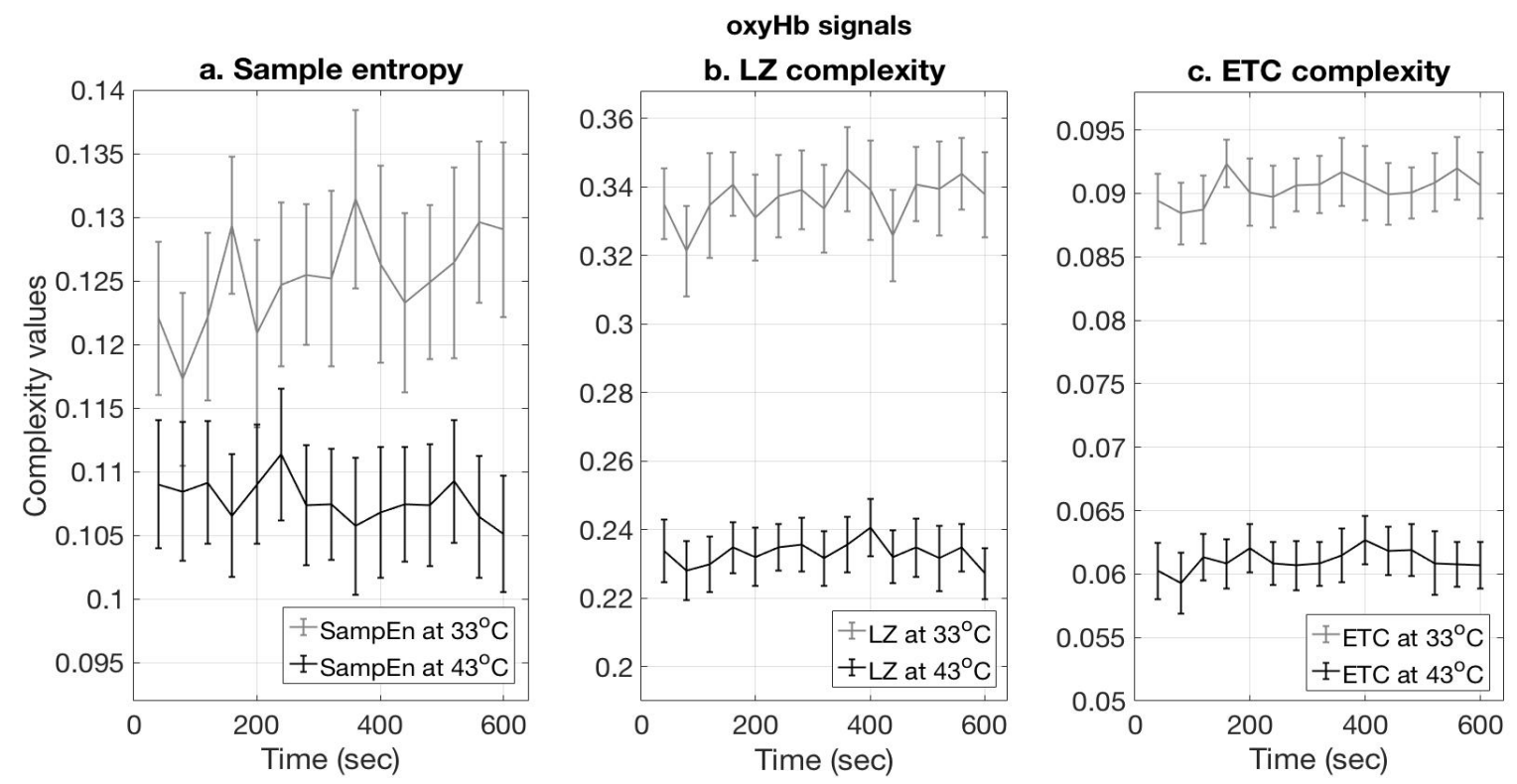

Fig. 6 Values of the complexity of the oxygenated haemoglobin signals for all 15 participants, in two haemodynamic steady states at $33^{\circ} \mathrm{C}$ (grey) and at $43^{\circ} \mathrm{C}$ (black). a) Sample Entropy, b) LZ complexity, c) Effort to compress complexity. Values are presented as mean \pm SEM.

The oxyHb signals showed a similar although more significant drop in LZ complexity $(p<0.001)$. Similar responses to the local heating were seen in the ETC complexity for BF and oxyHb signals showing a more substantial decline in the complexity at $43^{\circ} \mathrm{C}(p=0.002$, for $B F$ signals and $p<0.001$, for oxyHb signals).

Table 1 Mean, Standard deviation and mean standard error of all complexity methods at $33^{\circ} \mathrm{C}$ and at $43^{\circ} \mathrm{C}$ respectively for both $\mathrm{BF}$ and oxyHb signals $(n=15)$.

\begin{tabular}{|c|c|c|c|c|c|c|}
\hline \multirow[t]{2}{*}{ Temperature } & \multicolumn{3}{|c|}{$33^{\circ} \mathrm{C}$} & \multicolumn{3}{|c|}{$43^{\circ} \mathrm{C}$} \\
\hline & Mean & $\begin{array}{c}\text { Std. } \\
\text { Deviation }\end{array}$ & $\begin{array}{c}\text { Mean Std. } \\
\text { Error }\end{array}$ & Mean & $\begin{array}{c}\text { Std. } \\
\text { Deviation }\end{array}$ & $\begin{array}{l}\text { Mean Std. } \\
\text { Error }\end{array}$ \\
\hline \multicolumn{7}{|c|}{ BF signals } \\
\hline SampEn & 0.130 & 0.014 & 0.004 & 0.115 & 0.016 & 0.004 \\
\hline LZ complexity & 0.322 & 0.038 & 0.010 & 0.272 & 0.029 & 0.007 \\
\hline ETC complexity & 0.085 & 0.010 & 0.002 & 0.072 & 0.007 & 0.002 \\
\hline \multicolumn{7}{|c|}{ oxyHb signals } \\
\hline SampEn & 0.125 & 0.022 & 0.006 & 0.108 & 0.018 & 0.005 \\
\hline LZ complexity & 0.336 & 0.040 & 0.010 & 0.233 & 0.027 & 0.007 \\
\hline ETC complexity & 0.090 & 0.007 & 0.002 & 0.061 & 0.007 & 0.002 \\
\hline
\end{tabular}

Multiscale analysis of both $\mathrm{BF}$ and oxyHb signals in the two haemodynamic steady states across all 15 participants are shown in Fig. 7 and 8 respectively. The multiscale analysis of these signals showed a relatively compatible variability over the 24 scales at both temperatures. The $\mathrm{BF}$ and oxyHb parameters measured under the two haemodynamic steady conditions are summarized in Table 2. The results showed lower variability of the MSE of the $\mathrm{BF}$ and oxyHb signals during local heating compared with the signals at $33^{\circ} \mathrm{C}(p=0.001$, for $\mathrm{BF}$ signals and $p<0.001$, for oxyHb signals). 

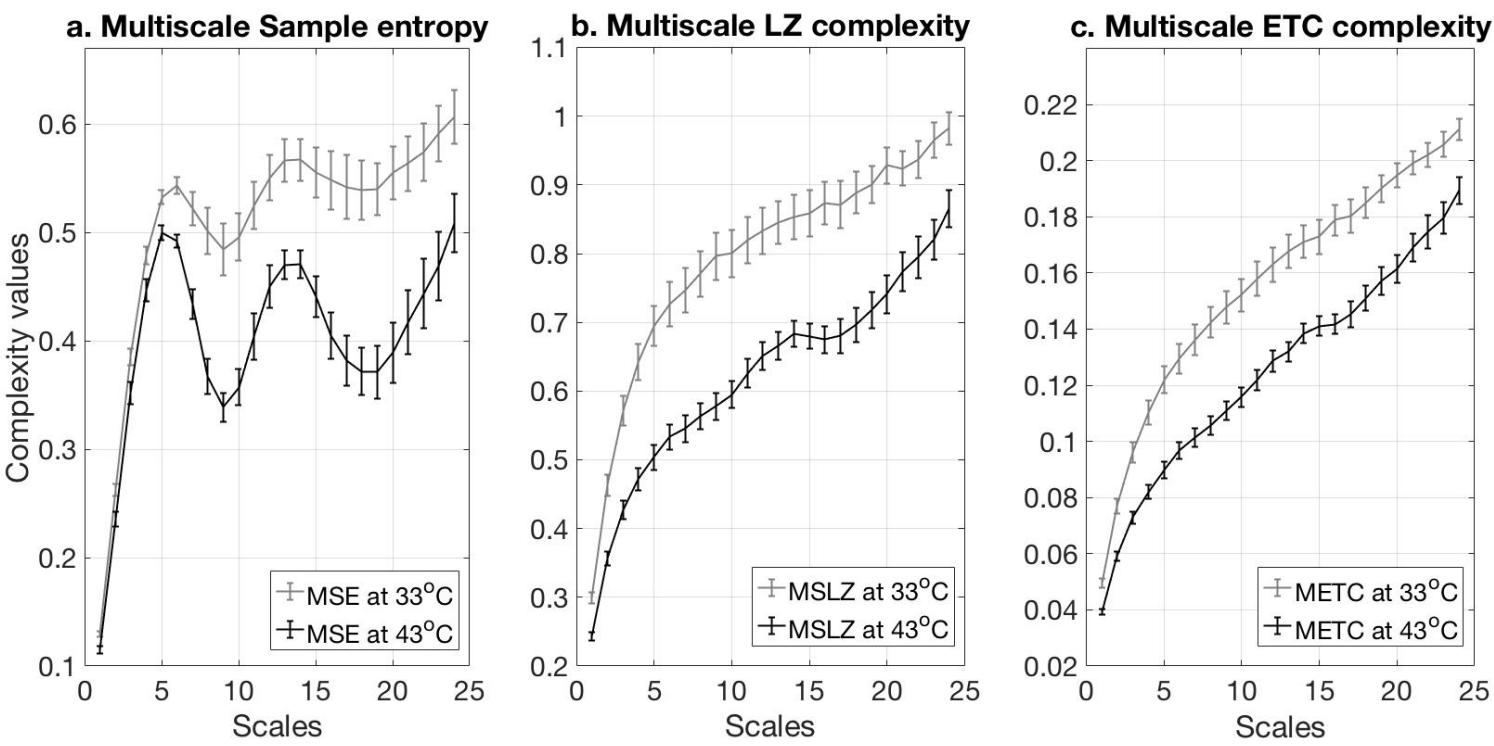

Fig. 7 Multiscale analysis for blood flow signals in two haemodynamic steady states at $33^{\circ} \mathrm{C}$ (grey) and at $43^{\circ} \mathrm{C}$ (black). a) Multiscale Sample Entropy, b) Multiscale LZ complexity, c) Multiscale Effort to compress complexity. Values are presented as mean \pm SEM of 15 healthy males.
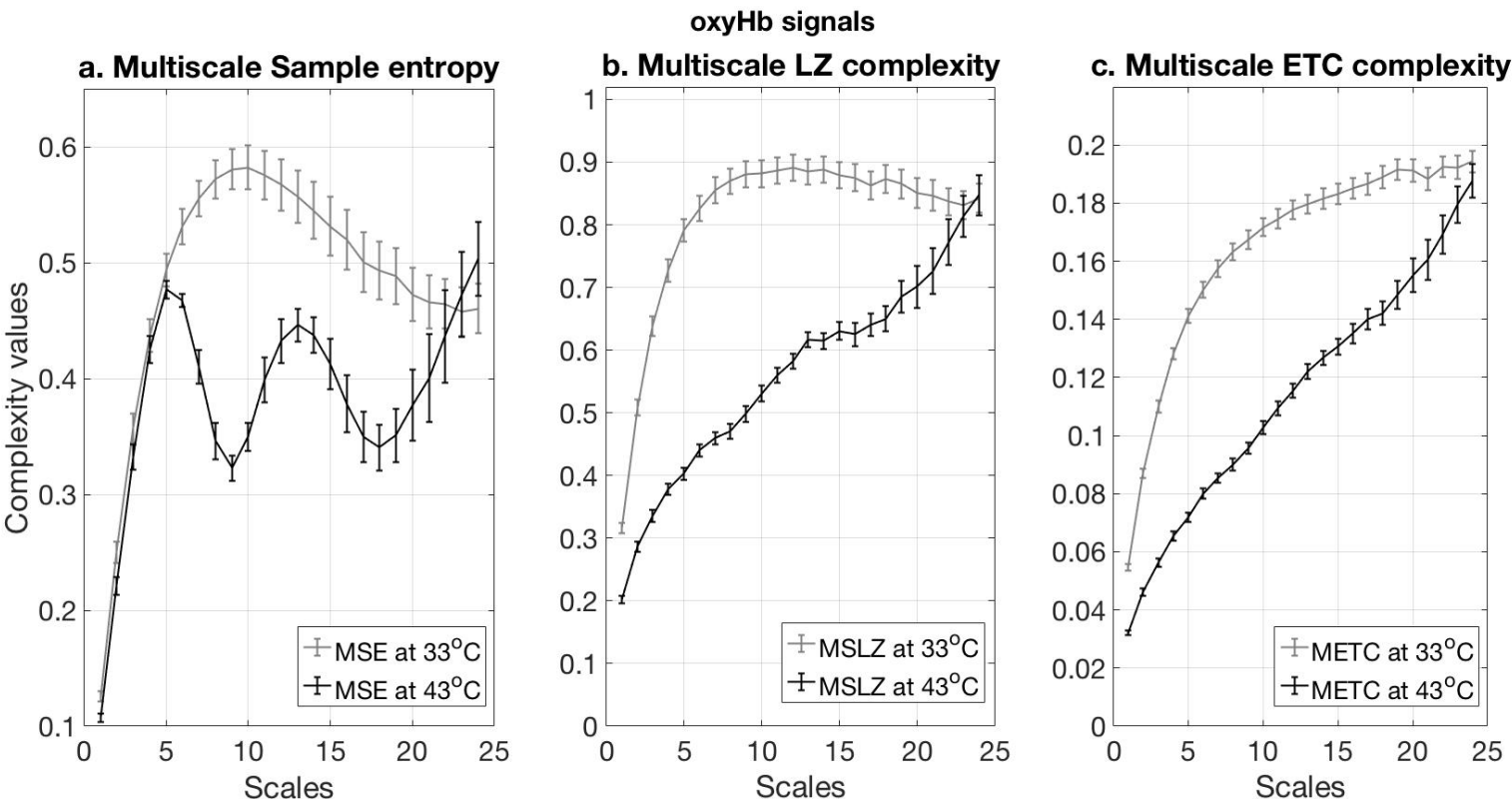

Fig. 8 Multiscale analysis for oxygenated haemoglobin signals in two haemodynamic steady states at $33^{\circ} \mathrm{C}$ (grey) and at $43^{\circ} \mathrm{C}$ (black). a) Multiscale Sample Entropy, b) Multiscale LZ complexity, c) Multiscale Effort to compress complexity. Values are presented as means \pm SEM of $n=15$ healthy males.

Similarly, the MSLZ and METC complexity values appeared less variable and had lower complexity of both $\mathrm{BF}$ and oxyHb signals at $43^{\circ} \mathrm{C}$, showing a considerable drop for both signals during LTH. Fig. 9 shows the boxplot of the conventional complexity and the multiscale complexity analysis for both $\mathrm{BF}$ and $\mathrm{oxyHb}$ signals showing the separation of the signals during LTH. It is shown that greater discrimination occurs in the oxyHb signals using the multiscale analysis $(p<0.001)$, as well as using the 
LZ and ETC complexity on a single scale. Similarly, for the multiscale analysis of the BF signals the separation was also good $(p=0.001)$. However, when using the conventional complexity analysis in $B F$ signals the separation between the haemodynamic steady states was shown to be lower than multiscale analysis or that found with the oxyHb signals ( $p>0.001$ ).

Table 2 Mean, Standard deviation and mean standard error of all multiscale analysis at $33^{\circ} \mathrm{C}$ and at $43^{\circ} \mathrm{C}$, respectively for both $\mathrm{BF}$ and oxyHb signals $(n=15)$.

\begin{tabular}{|c|c|c|c|c|c|c|}
\hline \multirow[t]{2}{*}{ Temperature } & \multicolumn{3}{|c|}{$33^{\circ} \mathrm{C}$} & \multicolumn{3}{|c|}{$43^{\circ} \mathrm{C}$} \\
\hline & Mean & $\begin{array}{c}\text { Std. } \\
\text { Deviation }\end{array}$ & $\begin{array}{l}\text { Mean Std. } \\
\text { Error }\end{array}$ & Mean & $\begin{array}{c}\text { Std. } \\
\text { Deviation }\end{array}$ & $\begin{array}{l}\text { Mean Std. } \\
\text { Error }\end{array}$ \\
\hline \multicolumn{7}{|c|}{ BF signals } \\
\hline MSE & 0.483 & 0.081 & 0.021 & 0.382 & 0.040 & 0.010 \\
\hline MSLZ & 0.765 & 0.141 & 0.037 & 0.589 & 0.087 & 0.022 \\
\hline METC & 0.151 & 0.025 & 0.007 & 0.119 & 0.015 & 0.004 \\
\hline \multicolumn{7}{|c|}{ oxyHb signals } \\
\hline MSE & 0.524 & 0.084 & 0.022 & 0.359 & 0.027 & 0.007 \\
\hline MSLZ & 0.848 & 0.090 & 0.023 & 0.526 & 0.057 & 0.015 \\
\hline METC & 0.169 & 0.013 & 0.003 & 0.107 & 0.011 & 0.003 \\
\hline
\end{tabular}
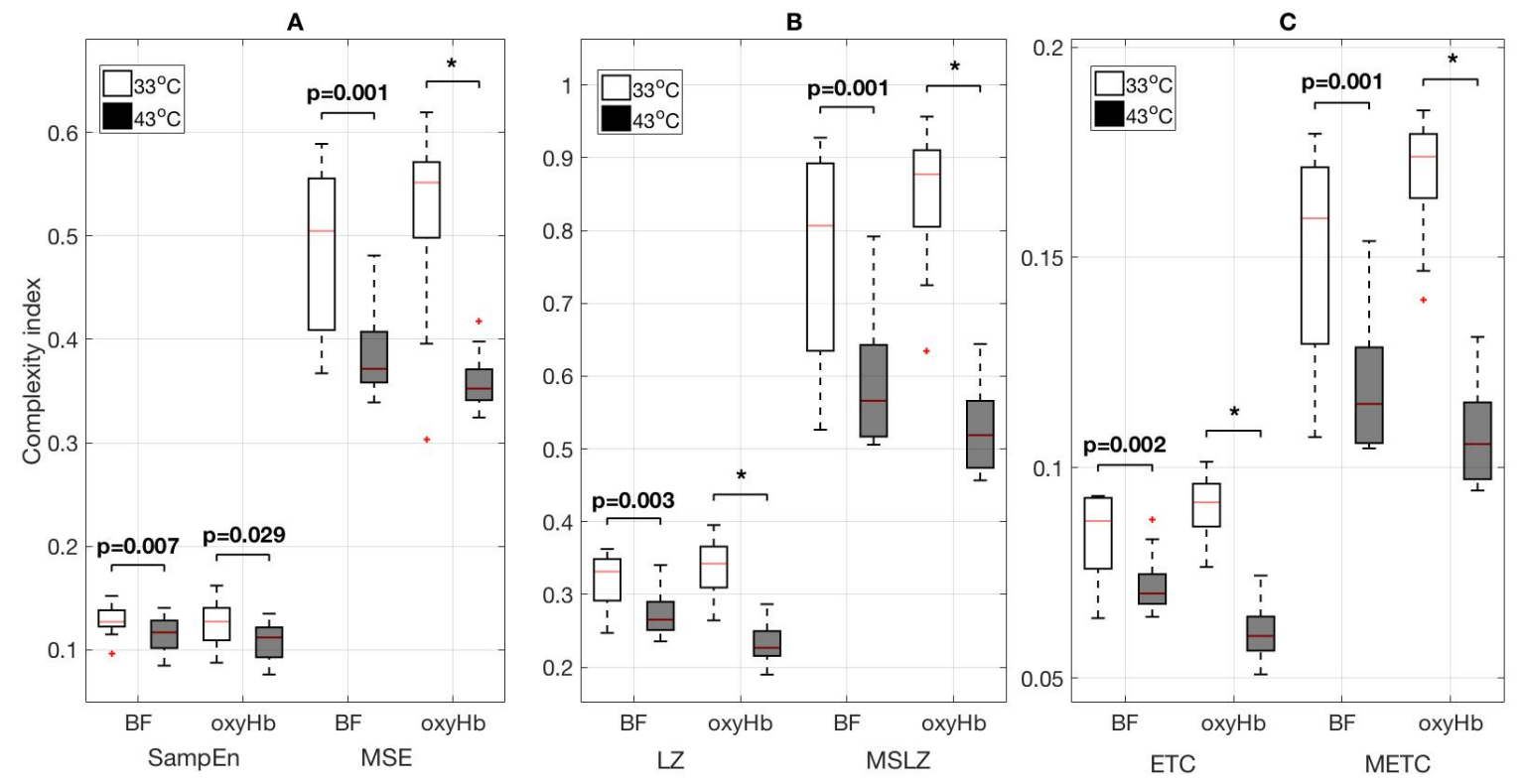

Fig. $9 \mathrm{BF}$ and oxyHb signals for all complexity and multiscale analysis expressed as mean value across 15 healthy male participants at $33^{\circ} \mathrm{C}$ (white) and at $43^{\circ} \mathrm{C}$ (black). A. Sample entropy and Multiscale Sample Entropy, B. LZ complexity and Multiscale LZ complexity, C. ETC complexity and Multiscale ETC complexity (“*” indicate $p<0.001$ ).

\section{Discussion}

We set out to investigate whether the information content in the BF and oxyHb signals derived from the skin microcirculation could be used to discriminate between two different haemodynamic states by examining the simultaneously recorded signals from a cohort of healthy male participants. 
The results show a significant decrease in the complexity of both signals during the vasodilated perfusion state at $43^{\circ} \mathrm{C}$. They further show that the multiscale analysis has the potential for distinguishing between the two different haemodynamic states in both $\mathrm{BF}$ and oxyHb signals, with a better discrimination in the oxyHb signals.

Recent studies [41, 42] have suggested that an increased variability in blood flux may indicate a more effective microvascular system, whereas a lower variability in microvascular activity corresponds to a loss of the system's ability to adapt under pathophysiological conditions. To our knowledge, no previous studies have investigated the SampEn and ETC complexity of healthy human skin blood flow and oxygenation signals, recorded simultaneously at the same site, during local heating. Our findings provide strong evidence that in healthy human skin microvascular blood flow and oxygenation signals, assessed using different complexity methods, showed sensitivity to the change in haemodynamic state induced by local warming. Here, lower complexity indexes with relatively consistent variation across the 15 epochs were found in all participants when microvascular flow approached maximum. By contrast, Liao et al. [15] investigating the sample entropy indexes in the sacral skin in people at risk for pressure ulcers of blood flow signals, found no significant correlation with the skin vasodilatory function during local warming. They also reported that the single scale sample entropy was not always a consistent method for the assessment of vasodilatory function concurring with our finding that sample entropy had lower classification reliability.

The traditional complexity methods measure the entropy and complexity of a signal only in one scale. For this reason, the $\mathrm{BF}$ and oxyHb signals were further examined using multiscale analysis, in order to evaluate these methods in multiple scales. At low scales, all the multiscale nonlinear methods (MSE, MSLZ and METC) yield small values of complexity. As the sampling rate decreases, the complexity increases until it reaches and passes the Nyquist frequency of the original time series. More specifically, by increasing the scale factor from $\tau=1$ to $\tau=12$, the sampling frequency which is $f_{s_{12}}=f_{s_{\text {original }}} / \tau=3.33 \mathrm{~Hz}$ reaches the Nyquist frequency which for our signals is $f_{N / 2}=2$. $f_{\text {max }}=2 \cdot 1.6 \mathrm{~Hz}=3.2 \mathrm{~Hz}$. We observed that for the complexity measures there is a rapid increase until the scale factor around 12 . At the higher scale factors, the complexity either decreases or becomes constant until scale factor around 20 . The complexity after the scale factor 20 steadily increases. However, multiscale sample entropy measures did not show the same changes through scales as the other two methods. Humeau et al. [20], in a recent study of the MSE analysis of LDF signals in healthy subjects, showed a similar behaviour of the MSE of the BF signal when filtered for the frequencies associated with heart rate $(\sim 0.6-2 \mathrm{~Hz})$. They reported that the increase and then decrease of the MSE over the scales may be because these signals were non-periodic and therefore the progression of complexity in multiple scales was not stable. They also suggested that this may be a representative indicator for the BF signals. It is also worth noting that during local warming around scale 24, the oxyHb signals become more complex (less regular) than those at $33^{\circ} \mathrm{C}$ using the MSE method and approaches that of those at $33^{\circ} \mathrm{C}$ using both MSLZ and METC complexity measures. This increase in complexity at higher scales may be a useful new index of increased adaptive capacity seen in the oxyHb signals at larger time scales possibly due to compensatory endothelial action.

The information of the signals during LTH using the multiscale analysis showed a similar decline to that when using the traditional methods. Here, we suggest that the multiscale analysis may be more valid than the traditional methods as it measures the complex characteristics of the biological signals in multiple spatial and temporal scales and is consistent with that reported by Kalev et al. [21], examining EEG signals for objective measures of depression. These authors found that traditional LZ complexity was unable to account for the high frequency components in the signals resulting in only a $50 \%$ classification accuracy of people with depression and controls in their study. Using multiscale LZ complexity, they were able to demonstrate an $86 \%$ classification accuracy by accounting for the 
different frequencies of information content in the EEG. In the current study, we were able to show $73.3 \%$ and $90 \%$ classification accuracy of haemodynamic state using MSLZ for BF and oxyHb, respectively [43].

All the nonlinear methods we used were able to distinguish between the two haemodynamic steady states. However, the LZ and ETC measures showed a more significant separation than the SampEn measure, in both conventional and multiscale analysis. Recently, Balasubramanian and Nagaraj [16] estimated the SampEn, LZ and ETC complexity in RR tachograms of healthy young and healthy old people in order to find the most efficient measure for a better separation between the two groups. They found that LZ and ETC complexity were able to discriminate better between the two groups for very short signal lengths in agreement with this study.

We found that, for the BF signals when using the multiscale analysis, there was a large difference between the two haemodynamic steady states, around scale factor 19 in the entropy indexes and around scale factor 9 in the complexity indexes. The weakest separation appears in scale 1 which is the scale studied by traditional entropy and complexity methods. For the oxyHb signals the strongest separation appears at around scale 9 for all methods. However, the smallest difference occurs at scale 4 in the entropy indexes and at scale 24 in the complexity indexes. It is also worth noting that the microvascular oxyHb signals showed better separation than the $\mathrm{BF}$ signals between the two haemodynamic steady states. This suggests that these measures may be valuable in clinical assessment in low perfusion states and in detecting early signs of sepsis. Other studies have also shown that pattern recognition methods are suitable for the discrimination between groups [44]. Pattern classification methods may be applied in future studies in order to classify the tissue perfusion features in different pathophysiological groups.

Previous studies [10], have shown that time and frequency domain analysis of the microvascular blood flow and oxygenation signals have not been able to produce definite and consistent interpretation of the microvascular function. It was also shown that the relative power spectral density contribution particularly in the low frequency bands was not significantly altered during vasodilation induced through $\mathrm{LTH}$ at $43^{\circ} \mathrm{C}$. The multiscale analysis used in this study showed that the complexity in lower time scales is lower and increases in larger scales indicating that the variability of the endothelial rhythm which is associated with microvascular dysfunction has a far longer time constant and would be better imaged at high scales. This suggests that nonlinear measures can be used to investigate the dynamics within the microcirculation and are able to provide a robust estimation in discriminating different pathophysiological conditions.

Recent studies [2], using chaotic network attractor analysis have shown that a declining adaptability in flow patterns in a microvascular network may be a major indicator of CV and Met disease risk. They also suggest that the spatial distribution and temporal behaviour of microvascular network perfusion may be more suitable measures with which to understand microvascular disease. More experiments need to be conducted in disease states to examine changes in complexity and for evaluating microvascular dysfunction.

\section{Limitation}

This study reveals the analysis of the information content of blood flow and oxygenation time series derived from the microvasculature in two haemodynamic steady states at $33^{\circ} \mathrm{C}$ and $43^{\circ} \mathrm{C}$ using both conventional and multiscale complexity methods. A major strengthen of this study is that by using LTH to assess microvascular reactivity and obtain two haemodynamic steady states, the nonlinear 
complexity methods were able to discriminate between the groups showing a better separation when using multiscale analysis. Our study seeks to explore the feasibility of using nonlinear complexity methods as a tool for differentiating between two imposed microvascular haemodynamic steady states as a proof of concept for clinical application in the prognosis and the diagnosis of pathophysiological conditions. However, this study was conducted only in healthy male cohorts as when including women in a study group it is essential to match them for menstrual cycle. We therefore elected to limit our study to young healthy males in order to reduce inter-individual variation as far as possible.

\section{Conclusion}

Here, we studied the nonlinear properties of the BF and oxyHb signals using SampEn, LZ and ETC complexity in order to identify the change of the vasodilation caused by local warming. We conclude that the LZ and ETC complexity measures show more sensitivity for identifying changes in the microvascular BF and oxyHb under an imposed stimulus than the SampEn measures. Our findings also suggest that the significant attenuation of the networks flexibility and adaptability in face of an imposed stressor, assessed using multiscale analysis of the complexity measures, makes this method a promising approach for further analysis of the microvascular function. For a better understanding of the nonlinear indexes of the microvascular function these methods now need to be extended to cohorts under pathological conditions.

\section{Acknowledgment}

We thank Katarzyna Kuliga for recruiting the study participants. We also thank Rodney Gush from Moor Instruments Ltd, Axminster, UK, for providing the LDF and OXY devices. This work was supported in part by the NIHR/Well come Trust Southampton Clinical Research Facility. M. Thanaj was supported by a UK EPSRC DTP PhD Studentship. The data for this work is accessible through the University of Southampton Institutional Research Repository at http://doi.org/10.5258/SOTON/D0343.

\section{References}

[1] M. Intaglietta, "Vasomotion and flowmotion: physiological mechanisms and clinical evidence," Vascular Medicine Review, vol. 1, pp. 101-112, 1990.

[2] J. C. Frisbee, J. T. Butcher, S. J. Frisbee, I. M. Olfert, P. D. Chantler, L. E. Tabone, A. C. d'Audiffret, C. D. Shrader, A. G. Goodwill, P. A. Stapleton, S. D. Brooks, R. W. Brock, and J. H. Lombard, "Increased peripheral vascular disease risk progressively constrains perfusion adaptability in the skeletal muscle microcirculation," Am J Physiol Heart Circ Physiol, vol. 310, no. 4, pp. H488-504, Feb 15, 2016.

[3] M. Costa, A. L. Goldberger, and C. K. Peng, "Multiscale entropy analysis of biological signals," Physical Review E, vol. 71, no. 2, Feb, 2005.

[4] J. C. Frisbee, A. G. Goodwill, S. J. Frisbee, J. T. Butcher, F. Wu, and P. D. Chantler, "Microvascular perfusion heterogeneity contributes to peripheral vascular disease in metabolic syndrome," J Physiol, vol. 594, no. 8, pp. 2233-43, Apr 15, 2016.

[5] A. Aldroubi, and M. Unser, Wavelets in Medicine and Biology: Taylor \& Francis, 1996.

[6] P. Flandrin, Time-Frequency/Time-Scale Analysis: Elsevier Science, 1998.

[7] J. L. Semmlow, Biosignal and Medical Image Processing, Second Edition: CRC Press, 2011.

[8] A. Stefanovska, M. Bracic, and H. D. Kvernmo, "Wavelet analysis of oscillations in the peripheral blood circulation measured by laser Doppler technique," leee Transactions on Biomedical Engineering, vol. 46, no. 10, pp. 1230-1239, Oct, 1999. 
[9] X. T. Tigno, B. C. Hansen, S. Nawang, R. Shamekh, and A. M. Albano, "Vasomotion becomes less random as diabetes progresses in monkeys," Microcirculation, vol. 18, no. 6, pp. 429-39, Aug, 2011.

[10] K. Z. Kuliga, R. Gush, G. F. Clough, and A. J. Chipperfield, "Time-dependent Behavior of Microvascular Blood Flow and Oxygenation: a Predictor of Functional Outcomes," IEEE Transactions on Biomedical Engineering, vol. PP, no. 99, pp. 1-1, 2017.

[11] P. Kvandal, A. Stefanovska, M. Veber, H. D. Kvenumo, and K. A. Kirkeboen, "Regulation of human cutaneous circulation evaluated by laser Doppler flowmetry, iontophoresis, and spectral analysis: importance of nitric oxide and prostaglandines," Microvascular Research, vol. 65, no. 3, pp. 160-171, May, 2003.

[12] M. Rossi, S. Bertuglia, M. Varanini, A. Giusti, G. Santoro, and A. Carpi, "Generalised wavelet analysis of cutaneous flow-motion during post-occlusive reactive hyperaemia in patients with peripheral arterial obstructive disease," Biomedicine \& Pharmacotherapy, vol. 59, no. 5, pp. 233-239, Jun, 2005.

[13] E. G. Salerud, T. Tenland, G. E. Nilsson, and P. A. Oberg, "RHYTHMICAL VARIATIONS IN HUMAN-SKIN BLOOD-FLOW," International Journal of Microcirculation-Clinical and Experimental, vol. 2, no. 2, pp. 91-102, 1983.

[14] A. Humeau, B. Buard, G. Mahe, D. Rousseau, F. Chapeau-Blondeau, and P. Abraham, "Multiscale entropy of laser Doppler flowmetry signals in healthy human subjects," Medical Physics, vol. 37, no. 12, pp. 6142-6146, Dec, 2010.

[15] F. Y. Liao, D. W. Garrison, and Y. K. Jan, "Relationship between nonlinear properties of sacral skin blood flow oscillations and vasodilatory function in people at risk for pressure ulcers," Microvascular Research, vol. 80, no. 1, pp. 44-53, Jul, 2010.

[16] K. Balasubramanian, and N. Nagaraj, "Aging and cardiovascular complexity: effect of the length of RR tachograms," Peerj, vol. 4, Dec, 2016.

[17] L. S. Correa, E. Laciar, V. Mut, B. F. Giraldo, A. Torres, and leee, "Multi-parameter Analysis of ECG and Respiratory Flow Signals to Identify Success of Patients on Weaning Trials," 2010 Annual International Conference of the leee Engineering in Medicine and Biology Society, IEEE Engineering in Medicine and Biology Society Conference Proceedings, pp. 6070-6073, 2010.

[18] F. Liao, W. D. O'Brien, Jr., and Y. K. Jan, "Assessing complexity of skin blood flow oscillations in response to locally applied heating and pressure in rats: implications for pressure ulcer risk," Physica A, vol. 392, no. 20, Oct 15, 2013.

[19] M. Costa, A. L. Goldberger, and C. K. Peng, "Multiscale entropy analysis of complex physiologic time series," Physical Review Letters, vol. 89, no. 6, Aug 5, 2002.

[20] A. Humeau, G. Mahe, F. Chapeau-Blondeau, D. Rousseau, and P. Abraham, "Multiscale Analysis of Microvascular Blood Flow: A Multiscale Entropy Study of Laser Doppler Flowmetry Time Series," leee Transactions on Biomedical Engineering, vol. 58, no. 10, pp. 2970-2973, Oct, 2011.

[21] K. Kalev, M. Bachmann, L. Orgo, J. Lass, H. Hinrikus, and leee, "Lempel-Ziv and Multiscale Lempel-Ziv Complexity in Depression," 2015 37th Annual International Conference of the leee Engineering in Medicine and Biology Society, IEEE Engineering in Medicine and Biology Society Conference Proceedings, pp. 4158-4161, 2015.

[22] V. E. Papaioannou, I. G. Chouvarda, N. K. Maglaveras, and I. A. Pneumatikos, "Temperature variability analysis using wavelets and multiscale entropy in patients with systemic inflammatory response syndrome, sepsis, and septic shock," Critical Care, vol. 16, no. 2, pp. 15, 2012.

[23] N. A. Strom, J. R. Sawyer, S. K. Roberts, S. M. Kingsley-Berg, and N. Charkoudian, "Local sensory nerve control of skin blood flow during local warming in type 2 diabetes mellitus," Journal of Applied Physiology (1985), vol. 108, no. 2, pp. 293-297, 2010. 
[24] K. Z. Kuliga, E. F. McDonald, R. Gush, C. Michel, A. J. Chipperfield, and G. F. Clough, "Dynamics of microvascular blood flow and oxygenation measured simultaneously in human skin," Microcirculation, vol. 21, no. 6, pp. 562-73, Aug, 2014.

[25] P. Kvandal, S. A. Landsverk, A. Bernjak, A. Stefanovska, H. D. Kvernmo, and K. A. Kirkeboen, "Low-frequency oscillations of the laser Doppler perfusion signal in human skin," Microvasc Res, vol. 72, no. 3, pp. 120-7, Nov, 2006.

[26] L. F. Liu, and S. X. Miao, "The complexity of binary sequences using logistic chaotic maps," Complexity, vol. 21, no. 6, pp. 121-129, Jul-Aug, 2016.

[27] X. S. Zhang, Y. S. Zhu, N. V. Thakor, and Z. Z. Wang, "Detecting ventricular tachycardia and fibrillation by complexity measure," leee Transactions on Biomedical Engineering, vol. 46, no. 5, pp. 548-555, May, 1999.

[28] M. Aboy, R. Hornero, D. Abasolo, and D. Alvarez, "Interpretation of the Lempel-Ziv complexity measure in the context of biomedical signal analysis," IEEE Trans Biomed Eng, vol. 53, no. 11, pp. 2282-8, Nov, 2006.

[29] Y. Zhang, S. Wei, H. Liu, L. Zhao, and C. Liu, "A novel encoding Lempel-Ziv complexity algorithm for quantifying the irregularity of physiological time series," Comput Methods Programs Biomed, vol. 133, pp. 7-15, Sep, 2016.

[30] A. C. Yang, S. S. Hseu, H. W. Yien, A. L. Goldberger, and C. K. Peng, "Linguistic analysis of the human heartbeat using frequency and rank order statistics," Phys Rev Lett, vol. 90, no. 10, pp. 108103, Mar 14, 2003.

[31] S. M. Pincus, "Approximate entropy as a measure of system complexity," Proceedings of the National Academy of Sciences of the United States of America, vol. 88, no. 6, pp. 2297-2301, 1991.

[32] J. S. Richman, and J. R. Moorman, "Physiological time-series analysis using approximate entropy and sample entropy," American Journal of Physiology-Heart and Circulatory Physiology, vol. 278, no. 6, pp. H2039-H2049, Jun, 2000.

[33] T. Skoric, O. Mohamoud, B. Milovanovic, N. Japundzic-Zigon, and D. Bajic, "Binarized crossapproximate entropy in crowdsensing environment," Computers in Biology and Medicine, vol. 80, pp. 137-147, Jan, 2017.

[34] H. Kantz, and T. Schreiber, Nonlinear Time Series Analysis, 2 ed., Cambridge: Cambridge University Press, 2003.

[35] A. M. Fraser, and H. L. Swinney, "Independent coordinates for strange attractors from mutual information," Physical Review A, vol. 33, no. 2, pp. 1134-1140, 02/01/, 1986.

[36] F. Kaffashi, R. Foglyano, C. G. Wilson, and K. A. Loparo, "The effect of time delay on Approximate \& Sample Entropy calculations," Physica D: Nonlinear Phenomena, vol. 237, no. 23, pp. 3069-3074, 2008/12/01/, 2008.

[37] D. Abasolo, C. J. James, and R. Hornero, "Non-linear analysis of intracranial electroencephalogram recordings with approximate entropy and Lempel-Ziv complexity for epileptic seizure detection," Conference proceedings : ... Annual International Conference of the IEEE Engineering in Medicine and Biology Society. IEEE Engineering in Medicine and Biology Society. Annual Conference, vol. 2007, pp. 1953-6, 2007, 2007.

[38] A. Lempel, and J. Ziv, "On the complexity of finite sequences," IEEE Trans. Inf. Theory, vol. IT22, no. 1, pp. 75-81, 1976.

[39] W. Ebeling, and M. A. Jiménez-Montaño, "On grammars, complexity, and information measures of biological macromolecules," Mathematical Biosciences, vol. 52, no. 1, pp. 53-71, 1980/11/01/, 1980.

[40] N. Nagaraj, K. Balasubramanian, and S. Dey, "A new complexity measure for time series analysis and classification," European Physical Journal-Special Topics, vol. 222, no. 3-4, pp. 847-860, Jul, 2013. 
[41] B. Gryglewska, M. Necki, M. Zelawski, M. Cwynar, T. Baron, M. Mrozek, and T. Grodzicki, "Fractal dimensions of skin microcirculation flow in subjects with familial predisposition or newly diagnosed hypertension," Cardiology Journal, vol. 18, no. 1, pp. 26-32, Jan, 2011.

[42] G. F. Clough, K. Z. Kuliga, and A. J. Chipperfield, "Flow motion dynamics of microvascular blood flow and oxygenation: Evidence of adaptive changes in obesity and type 2 diabetes mellitus/insulin resistance," Microcirculation, vol. 24, no. 2, 2017.

[43] M. Thanaj, A. J. Chipperfield, and G. F. Clough, "Multiscale Analysis of Microvascular Blood Flow and Oxygenation," World Congress on Medical Physics and Biomedical Engineering 2018. pp. 195-200.

[44] M. Costa, and J. A. Healey, "Multiscale entropy analysis of complex heart rate dynamics: Discrimination of age and heart failure effects," Computers in Cardiology 2003, Vol 30, Computers in Cardiology A. Murray, ed., pp. 705-708, New York: leee, 2003. 